\title{
UN ESTUDIO DE LA DISCIPLINA PARTIDISTA DE LOS DIPUTADOS ESPAÑoles EN EL CoNGReso
}

\section{A Study of Party Discipline in the Spanish Congress of Deputies}

\author{
GEMA SÁNCHEZ MEDERO \\ Universidad Complutense de Madrid, España
}

\section{BERNABÉ ALDEGUER CERDÁ}

Universidad de Alicante, España

\begin{abstract}
RESUMEN
En este artículo se examinan las relaciones que mantienen en España, los partidos políticos, grupos parlamentarios y diputados en el Congreso de los Diputados, con el objetivo de medir el grado la disciplina existente. Pero, a diferencia de otros estudios que se han efectuado sobre esta cuestión, el análisis no se va a limitar a explorar las medidas disciplinarias de manera descriptiva, sino que, además, se van ofrecer otro tipo de herramientas de carácter cuantitativo que sirven para evaluar el grado de disciplina. Gracias a ello, se obtendrá una información crucial sobre cuál es la naturaleza de la disciplina, cuáles son los beneficios y los costos que tienen para los parlamentarios actuar colectiva o individualmente, qué factores inciden en los niveles de cohesión y disciplina, además de cómo se pueden determinar los mismos.
\end{abstract}

Palabras clave: disciplina partidista, grupos parlamentarios, diputados, parlamento, cohesión, España

\begin{abstract}
This paper analyses relations between political parties, parliamentary groups, and members of the Chamber of Deputies, with the aim of measuring the extent of existing party discipline. Unlike other studies that have examined this issue, the current analysis is not limited to descriptive discussions of disciplinary measures, but uses quantitative tools in order to assess the extent of parliamentary discipline. As a result, we obtain crucial information on the nature of party discipline, the costs and benefits that parliamentarians face when acting individually versus collectively, and the factors that affect levels of cohesion and discipline and how we determine them.
\end{abstract}

Keywords: parliamentary discipline, parliamentary groups, deputies, parliament, cohesion, Spain 


\section{INTRODUCCIÓN}

Las relaciones entre los diputados y los grupos parlamentarios obedecen a dos cuestiones básicas: la afiliación y la integración en las listas electorales (Morales 1990). Los congresistas se someten a una doble disciplina: la propia del grupo en el que desarrollan su actividad y la del partido en el que militan. De esta manera, el diputado recibe un doble mandato: el del elector y el de su partido (Guerrero Salom 2004: 96), como consecuencia de la controvertida relación que se produce entre la atribución representativa y aquella imperativa. El problema reside en que, en caso de conflicto entre estos, el parlamentario se optará por el segundo en detrimento del representativo para no arriesgar su reelección. Por tanto, los parlamentos funcionan - a todos los efectos- como una "cámara de partidos". Tal es así que, actualmente, las democracias no son solo parlamentarias, sino que también son partidistas.

Son muchos las investigaciones que tratan de explicar las relaciones que se entablan entre los partidos políticos y sus grupos parlamentarios, así como entre los diputados y sus bancadas. La cuestión es que un buen número de ellas se han centrado en las reformas operadas por los reglamentos partidistas y parlamentarios, en los pronunciamientos jurisprudenciales que profundizan entorno a esa dependencia, en la unidad del voto (Depauw y Martin 2009), en las medidas disciplinarias - principalmente atendiendo a estudios de caso en el Reino Unido, Alemania y el Norte de Europa (Lees y Shaw 1979; Arter 1984; Damgaard 1992) - o a estudios comparativos de carácter descriptivo (Hazan 2003). Pero son pocas las que han tenido como objeto aquellos mecanismos que conducen a la cohesión del grupo del partido en el Parlamento (Saalfeld y Becker 2000) y, prácticamente, son inexistentes las que intentan demostrar cómo esos factores indicen realmente en la disciplina parlamentaria, quedando, en el mejor de los casos, en un mero marco descriptivo. La razón puede deberse a la dificultad que entraña un análisis como este, ya que la disciplina es un fundamento oculto, no explícito formalmente, del gobierno de los partidos en el régimen parlamentario. Tal es así, que esta se encuentra al margen de las propias normas de cualquier cámara de representantes.

Por tal motivo, en este artículo se intentará hacer el esfuerzo de aportar algo más que la descripción normativa, por lo cual se van ofrecer otro tipo de herramientas que sirven también para medir el grado de disciplina. Por supuesto, no se va a descuidar este aspecto normativo, que es esencial para conocer el funcionamiento formal de cualquier organización, sino que también se va hacer alusión a otras cuestiones de carácter cuantitativo e informal. Además, gracias a la unión de ambas perspectivas de análisis, se podrá obtener una información crucial sobre cuál es la naturaleza de la disciplina, cuáles son los beneficios y costos que tienen para los parlamentarios cuando actúan colectiva o individualmente, qué factores inciden en los niveles de cohesión y disciplina y cómo se puede medir las mismas. 
El hecho de seleccionar la cámara baja española se debe a que estas cuestiones pueden ser analizadas mejor en esta arena parlamentaria dado el alcance de su reglamento a este respecto. Téngase en cuenta que la disciplina ha jugado un papel fundamental en el proceso de construcción y consolidación de la democracia en España, un hecho característico de su transición política. Además, aquí los partidos son básicamente estructuras parlamentarias, ya que la normativa española, a diferencia de otras, pretende convertir a los grupos en pseudoinstrumentos de los partidos políticos. Por tanto, estamos ante un parlamento donde la actividad del diputado está mediatizada por el partido y su posición se encuentra definida por la subordinación del criterio individual al parecer general del partido, como consecuencia de la disciplina de grupo.

Como observamos, todo un caso es este español paradigmático de la disciplina de partidos en la Cámara Baja. Por lo mimo que nos permite obtener una información relevante sobre las complejas relaciones entre parlamentarios, grupos y partidos en los sistemas parlamentarios y, por tanto, ofrece una radiografía de cómo funciona realmente el parlamento en este tipo de sistemas políticos. De ahí que se haya optado por examinar este aspecto haciendo especial referencia en las actividades que los congresistas efectúan en la cámara y a la disciplina a la que se ven sometidos en el ejercicio de sus funciones, junto con las consecuencias que acarrea el irresuelto problema de la prohibición del mandato imperativo. Aunque únicamente se han tomado como objeto de estudio los dos grupos parlamentarios más importantes y que han estado presentes en todas las legislaturas en el Congreso de los Diputados —el Partido Popular (PP) y el Partido Socialista Obrero Español (PSOE)—, esto no nos impide hacer alusión a otros grupos parlamentarios y ejemplos significativos. Gracias a ello, los resultados no solo servirán para comprender la verdadera naturaleza de la cohesión de los parlamentarios, sino que además podrán servir como guía para otras investigaciones, al ofrecer nuevas pistas sobre cómo se vertebra la disciplina parlamentaria.

\section{LA DISCIPLINA PARTIDISTA}

La literatura que aborda el tema de la disciplina partidista se ha empeñado en proponer distintas maneras de definir a los partidos políticos para explicar el comportamiento de los diputados (González Tule 2010). En términos conceptuales existen, básicamente, dos grupos de análisis: primero, la diferencia entre disciplina y cohesión partidista y, segundo que trata ambos conceptos indistintamente, sin distinción alguna (Ozbudun 1970; Bowler et al. 1999).

Así, para unos, la disciplina es resultado de las relaciones de poder en el interior de los partidos políticos, impuesta desde arriba y enmarcada por la distribución de sanciones e incentivos, mientras que la cohesión refleja el grado de coordinación entre los diputados ante la necesidad de competir electoralmente, sin que sea necesario implantar medidas disciplinarias (Laver y Shepsle 1999: 
29). Por tanto, la apelación a la disciplina se da cuando la cohesión es baja y no es necesaria cuando esta es alta (Heller y Mershon 2002). De esta manera, cuando la cohesión se debilita es cuando la disciplina puede y, en la mayoría de los casos, debe ayudar a mantener la unidad del partido (Lacina 2009: 4). Para los segundos, disciplina y cohesión resulta ser lo mismo.

En todo caso, para abordar el tema de la disciplina es pertinente señalar que esta se basa en la autodisciplina — que es la que los integrantes de un grupo parlamentario se imponen a sí mismos- y la heterodisciplina, que es la que es fijada por los dirigentes del partido. Según Giovanni Sartori (1999), cuando la segunda opción supera a la primera, la asamblea se transforma en una mera caja de resonancia de decisiones elaboradas en el exterior. Y es cuando la Cámara debe garantizar el equilibrio entre los mecanismos institucionales que favorecen la disciplina partidista y aquellos que aseguran que los representantes de los ciudadanos deben responder a las demandas del electorado (responsiveness) y asumir las responsabilidades (accountability) por sus acciones (Béjar 2002). Otra cuestión es que se consiga esto, porque en el seno de los parlamentos, se suele introducir una férrea estructura jerárquica que descansa en la subordinación del diputado a su jefe de bancada y éste a la de su partido y a la de su líder (Aragón Reyes 2008: 138). Por tanto, la disciplina partidista en la Cámara es entendida como el acatamiento de los miembros de un partido (independientemente de sus preferencias individuales) a las instrucciones o a la influencia de su líder (Bowler et al. 1999).

El argumento que se esgrime es que la disciplina beneficia al sistema político (Béjar 2002) ya que permite obtener los votos necesarios para aprobar las iniciativas y, de esta manera, evitar el bloqueo institucional (Holgado 2016). Pero también puede ser perjudicial cuando se tiende a desplazar el interés de los electores en beneficio exclusivo de los partidos o cuando estos controlan la formación de mayorías y se oponen sistemáticamente a las propuestas del ejecutivo.

Llegados a este punto, habría que preguntarse ¿cómo el grupo parlamentario consigue disciplinar a sus diputados en un sistema parlamentario? La respuesta obliga a hacer una reflexión desde distintas perspectivas:

1. Las líneas sobre las que gira el diseño constitucional y el sistema electoral (Cox et al. 2009). Por ejemplo, se presupone que los sistemas parlamentarios favorecen la constitución de partidos más disciplinados quelos presidenciales (Ozbudun 1970) o las listas cerradas y bloqueadas hacen más dependientes a los diputados de sus partidos (Carey 2007) ya que los electores no tienen capacidad para influir en la composición de las mismas y los representantes no tienen que ganarse el puesto ante ellos. Asimismo, los representantes elegidos a través de los sistemas proporcionales son más propensos a reaccionar a las medidas disciplinarias (Stratmann y Baur 2002), etcétera. 
2. Los líderes de los partidos políticos controlan los mecanismos de nominación, así como los recursos para premiar o castigar a los diputados. Sin duda, ellos también contribuyen en los sistemas parlamentarios a mantener la disciplina partidista (Holgado 2016).

3. La constante rotación de los cargos permite a los dirigentes de los partidos una mayor intervención en el proceso de selección de candidatos, desplazando así el poder e influencia de los votantes (Nacif 2000).

4. Los dirigentes de los grupos, en amparo de las distintas normas, pueden controlar, casi cualquier actividad de los diputados en la Cámara.

5. La propia coyuntura y la que integra los temas en la agenda política, pero son los portavoces de los grupos los que determinan las iniciativas que serán discutidas y votadas por los grupos parlamentarios en el Pleno y las comisiones.

\section{METODOLOGÍA}

Este estudio se desarrolla en base a una hipótesis inicial de trabajo: el alto grado de dependencia que tienen los diputados con el grupo parlamentario al que se adscriben y pertenecen, como consecuencia de los propios estatutos de los partidos políticos, así como del ordenamiento jurídico y reglamentario de carácter parlamentario, el sistema electoral y las propias dinámicas que en la práctica promueven sus derechos y deberes.

En concreto, las hipótesis planteadas en esta investigación son:

H1. Cuanta más influencia tienen los votantes a la hora de determinar la composición de la cámara, menor es la capacidad de los partidos y del grupo para hacer prevalecer la disciplina sobre sus diputados.

H2. Cuanto más centralizado es el proceso de selección de candidatos, mayor será la fuerza de las medidas disciplinarias entre los parlamentarios.

H3. Cuanto más grande es un grupo parlamentario, más intensas serán las medidas disciplinarias.

H4. Cuantos más incentivos deseen obtener los diputados para impulsar su carrera política, mayor será la fuerza que tendrán las medidas disciplinarias del grupo parlamentario sobre ellos.

Con objeto dedemostrar lashipótesis esbozadas, sehan empleado,comounidades de análisis: 1) la normativa existente a nivel de partido (ej. los estatutos de los partidos políticos), 2) la regulación parlamentaria (el Reglamento del Congreso de los Diputados y los reglamentos internos de los grupos parlamentarios) y, 3) la Constitución española y la legislación electoral, (v. gr., Ley Orgánica del Régimen Electoral (LOREg) o la Ley Orgánica de Partidos Políticos). Se debe 
considerar, de todas formas, que la parte central del estudio versa sobre la normativa interna de los propios partidos y grupos parlamentarios, porque son en este tipo de documentos donde aparece recogido el verdadero carácter de la relación entre partidos, grupos y diputados. No obstante, también se ha recurrido a otras fuentes como los reglamentos parlamentarios y la normativa estatal, con el objetivo de corroborar un hecho particular del ordenamiento español, como le es el reconocimiento explícito que hace de la imbricación entre el partido y el grupo, debilitando con ello la figura del representante individual.

Además, este análisis se ha visto complementado con la realización de una serie de entrevistas a una serie de diputados relevantes de ambos grupos parlamentarios. En concreto, se realizaron 16 entrevistas, ocho de las cuales fueron a diputados del Grupo del PSOE (GPSOE) y ocho a de diputados del grupo del PP (GPP), de los cuales tres pertenecían a los órganos de dirección del grupo y 13 al pleno. Las preguntas se refirieron a las siguientes dimensiones: a) los instrumentos institucionales y b) los instrumentos procesuales (Raunio 2000). Además, se ha utilizado el Estudio 2827 del Centro de Investigaciones Sociológicas (CIS), en el cual se aborda una investigación sobre las élites políticas en España en toda su dimensión. Gracias al mismo, se ha podido verificar cómo se adoptan las decisiones en los grupos parlamentarios, el grado de autonomía de los diputados, los criterios que priman a la hora de seleccionar a los miembros que van integrar las listas electorales y las opiniones de los diputados a este respecto. Asimismo, se ha empleado el estudio de Guillermo Cordero y Xavier Coller (2013) sobre la selección de candidatos y la disciplina del partido para tomar los datos de los índices de centralidad en el proceso de la elaboración de las listas electorales.

Por otra parte, se debe advertir que, si únicamente se ha optado por centrar el estudio en la Cámara Baja y en los dos grandes grupos parlamentarios, ha sido principalmente por tres razones: primero, porque los partidos a nivel autonómico se han dedicado a reproducir las mismas pautas organizativas que las agrupaciones a nivel nacional. Pese a que se les reconoce total autonomía para dotarse de las normas que van a venir a regular su funcionamiento, estas estructuras organizativas guardan casi total similitud con las de la organización nacional. Segundo, España cuenta con 17 parlamentos autonómicos y cada uno de ellos dispone de su propio reglamento parlamentario, ampliando en exceso nuestro objeto de estudio, más aún cuando en el ordenamiento parlamentario autonómico español, la naturaleza "grupo-crática" se hace todavía más evidente. Finalmente, en los grupos parlamentarios grandes se ponen de manifiesto, más explícitamente, las medidas para garantizar la disciplina interna. 


\section{LA ACTIVIDAD DE LOS DIPUTADOS NACIONALES EN EL GRUPO PARLAMENTARIO Y EN LA CÁMARA}

El Reglamento del Congreso de los Diputados (RCD) tiende a proclamar la preeminencia del diputado en la Cámara pero, en la práctica, no es así. La mayoría de los reglamentos internos de las bancadas parlamentarias sostienen que son los Plenos del grupo los que adoptan la posición del mismo en el desarrollo de su actividad parlamentaria. Por ejemplo, el Reglamento del Grupo Popular (RGPP) indica que es el Pleno del grupo donde se debaten, principalmente, las propuestas que realiza el Consejo de Dirección del grupo (órgano de dirección) para fijar los criterios de actuación del mismo en la Cámara. Además, gracias a las entrevistas ${ }^{1}$ que se han efectuado a los diputados, se sabe que solo cuando exista una cuestión que pueda crear ciertas diferencias, incluso de posición, el Pleno del grupo se convierte en un verdadero órgano deliberante y decisorio.

En cambio, el Reglamento del Grupo Parlamentario Socialista (RGPSOE) entiende, en principio, que en el Pleno del grupo se podrán debatir cualquier asunto que sea propuesto por sus miembros, incluso se abre la posibilidad a que diez diputados puedan alterar la orden del día de las sesiones ordinarias. Aunque, en definitiva, es el Comité Director a quien le corresponde la dirección organizativa y política del grupo, la autorización de las iniciativas propuestas por los parlamentarios socialistas y la designación de los miembros del grupo en las comisiones de la Cámara, es decir, el órgano de dirección del grupo parlamentario (art. 18. RGPSOE).

Tabla 1. Órganos de los grupos parlamentarios popular y socialista

\begin{tabular}{ll}
\hline \multicolumn{1}{c}{ GPP } & \multicolumn{1}{c}{ Composición y funciones } \\
\hline Consejo de dirección & $\begin{array}{l}\text { Presidente, Secretario General, Portavoz, Secretario General } \\
\text { del PP, Coordinador de Comisiones Parlamentarias, portavo- } \\
\text { ces adjuntos, Tesorero, Tesorero del PP, miembros del Grupo } \\
\text { en la Mesa del Congreso, Jefe de la Asesoría Parlamentaria y } \\
\text { Secretario Técnico. Su función es la dirigir el grupo, establecer } \\
\text { los criterios a seguir en el grupo y garantizar la disciplina. } \\
\text { Todos los miembros del grupo parlamentario. Su función es } \\
\text { debatir y acordar la postura del grupo parlamentario en la } \\
\text { Cámara. } \\
\text { Pleno } \\
\text { Sus funciones son la dirección, la coordinación y el control } \\
\text { de todos los trabajos que realice el grupo y los servicios téc- } \\
\text { nicos y administrativos del mismo. } \\
\text { Especialistas en derecho. Su función es asesorar al grupo y a } \\
\text { los diputados en su quehacer en la Cámara. }\end{array}$ \\
\hline
\end{tabular}

Fuente: elaboración propia 
Por tanto, son los órganos de dirección de los grupos los que toman las decisiones, ya que son los que tienen la última palabra. Este hecho viene a corroborar los datos de la Tabla 2, según la cual, en los dos grupos parlamentarios, más del $60 \%$ de las decisiones que se adoptan son consecuencia de la disciplina del partido y solo una parte relativamente pequeña fruto de la deliberación en el Pleno. Estos datos contrastan con los de "otros partidos", ya que en esos, la deliberación en el grupo presenta unos mayores porcentajes. Ello confirma la tercera hipótesis, pues el grado de disciplina interna es mayor en los grupos más numerosos que en los pequeños. En este sentido, no se puede obviar que en los grandes grupos parlamentarios existen más opiniones y, por lo tanto, se requiere un mayor esfuerzo de coordinación (Carey 2007).

Pero la escasa autonomía que poseen los miembros del grupo para tomar decisiones, también queda reflejada en la segunda parte de la Tabla 2. Más del $80 \%$ de los diputados aceptan la disciplina del partido, aunque eso sí, suelen expresar su opinión. Pero el dato más relevante es que más de un 12\% mantienen una postura de apoyo firme a la disciplina y siempre votan siguiendo la dirección que marque la cúpula del grupo. Solo los diputados de la Izquierda Unida (IU) presentan porcentajes algo más elevados que las otras dos fuerzas políticas a la hora de ignorar dicha disciplina. Esta unidad del voto implica, pues, el interés que muestran los diputados por continuar su carrera política en la Cámara, dado que es conocido por todos que quienes deciden votar con arreglo a su conciencia son sancionados y, si repiten dicho comportamiento, corren el riesgo de no formar parte de las listas electorales en los siguientes comicios, más cuando estas son cerradas y bloqueadas. Ello confirma la primera y la tercera hipótesis.

Tabla 2. Toma de decisiones en los grupos parlamentarios (en porcentajes)

\begin{tabular}{lccc}
\hline & $\begin{array}{c}\text { Disciplina } \\
\text { del partido }\end{array}$ & $\begin{array}{c}\text { Delibera- } \\
\text { ción }\end{array}$ & Total \\
\hline PP diputados nacionales & 66 & 34 & 100 \\
Psot diputados nacionales & 61 & 39 & 100 \\
Otros partidos & 49 & 51 & 100 \\
Total nacional & 63 & 37 & 100 \\
& PSOE & PP & IU \\
Hacer constar su opinión pero aceptar la dis- & 80 & 81 & 69 \\
ciplina del partido & 11 & 14 & 5 \\
Votar con su partido & 2 & 2 & 5 \\
Abstenerse & 4 & 1 & 10 \\
Ignorar la disciplina del partido y votar inde- & & & \\
pendientemente & & & 5 \\
\hline
\end{tabular}


Por tanto, no es de extrañar que el índice de Rice se sitúe en 99\% ante la alta cohesión que muestran los partidos españoles cuando votan en el Congreso de los Diputados (Field 2016). Hecho que se ve confirmado por los datos de la Tabla 3, según los cuales todos los partidos presentan unos elevados índices de disciplina, principalmente los diputados socialistas, populares y nacionalistas gallegos. Esto demuestra que el voto en conciencia solo se produce en contadas ocasiones y no de manera sistemática.

No obstante, hay que señalar que estos altos índices de disciplina no son hechos aislados del caso español, sino que también se producen en una buena parte de las democracias parlamentarias europeas, donde el índice Rice se encuentra por encima del 95\% (Carey 2000). Otra cosa distinta es lo que sucede en los sistemas presidencialistas - como EE. UU. o las repúblicas latinoamericanas-, donde los partidos parlamentarios no tienen por qué sostener al gobierno y, por tanto, la indisciplina y el voto independiente son más frecuentes, y esto conduce a que el índice de unidad oscile entre el 70\% y el 80\% (Carey 2000). La razón de esta diferencia estriba en el distinto nivel de protagonismo que adquieren los partidos políticos en la vida parlamentaria en uno u otro sistema, es decir, mientras que en los sistemas presidencialistas los partidos juegan un papel subsidiario a la hora de dirigir el posicionamiento de los diputados, en los regímenes parlamentarios los partidos desempeñan un rol determinante.

Tabla 3. Índice de acuerdo (IA) promedio de las legislaturas V, VI, VIII y IX

\begin{tabular}{|c|c|}
\hline Partidos & Índice \\
\hline PSOE & 99,2 \\
\hline PP & 98,7 \\
\hline IU & 98,4 \\
\hline CIU & 98,2 \\
\hline PNV & 98,2 \\
\hline CC & 94,5 \\
\hline ERC & 98,7 \\
\hline BNG & 99,6 \\
\hline
\end{tabular}

Fuente: Field (2016)

$\mathrm{IA}=$ votos a favor $-($ votos en contra + abstenciones $) /$ votos totales

No obstante - y pese a estos datos-, la mayoría de los diputados considera que disfrutan de autonomía para desempeñar sus funciones en la Cámara, pero, eso sí, siempre es conveniente consultar a los dirigentes antes de tomar cualquier decisión. Solo unos pocos creen que poseen una total autonomía, principalmente los diputados de IU, pero siempre que respeten las directrices generales del grupo y del partido, de manera que no cabe duda que se está produciendo un debilitamiento del concepto de representación. Este hecho se ve reforzado por el sistema electoral imperante, donde las listas cerradas y bloqueadas hacen que 
el diputado busque antes el apoyo del partido que el de sus electores (Carey y Shugart 1995: 430). Con ello, el tamaño del grupo y la escasa capacidad de los electores para determinar la composición de la Cámara vuelve a ser relevante para establecer el grado de disciplina.

Tabla 4. Autonomía de los diputados

\begin{tabular}{lccccc}
\hline Totalmente & $\begin{array}{c}\text { Tienen auto- } \\
\text { nomía, aunque } \\
\text { hay unas } \\
\text { directrices } \\
\text { autónomos } \\
\text { generales a las } \\
\text { que hay que } \\
\text { ajustarse }\end{array}$ & $\begin{array}{c}\text { Tienen auto- } \\
\text { nomía, pero } \\
\text { conviene que } \\
\text { consulten con } \\
\text { los dirigentes } \\
\text { antes de tomar } \\
\text { una decisión }\end{array}$ & $\begin{array}{c}\text { Tiene poco } \\
\text { autonomía, } \\
\text { porque la posi- } \\
\text { ción del grupo } \\
\text { les suele venir } \\
\text { dada }\end{array}$ & $\begin{array}{c}\text { Generalmente } \\
\text { no tienen auto- } \\
\text { nomía }\end{array}$ \\
\hline PP & 5 & 29 & 43 & 13 & 10 \\
PSOE & 1 & 28 & 44 & 17 & 8 \\
IU & 12 & 47 & 23 & 12 & 6 \\
Otros & 11 & 45 & 30 & 9 & 5 \\
\hline
\end{tabular}

Fuente: base de datos del Centro de Investigaciones Sociológicas (CIs), Estudio 2827

Para continuar profundizando en esta cuestión, es necesario analizar la relación existente entre los diputados y su grupo a la hora de desempeñar su actividad dentro de la Cámara. En este sentido, hay que señalar que el órgano que nombra a los diputados que van acudir a cada Comisión es el Portavoz del grupo parlamentario, el que decide qué iniciativas van a ser defendidas y por quién, mientras que es designado por el órgano directivo del partido. De esta manera, se trata de evitar que se puedan presentarse propuestas que vayan a contradecir las líneas políticas del grupo y del partido, más cuando son las direcciones de los grupos las que determinan los criterios y las directrices de actuación. ${ }^{2}$ Si esto no fuera suficiente, el RCD no hace más que condicionar la libertad de actuación de los diputados cuando exige: 1) que todas las enmiendas sean firmadas por el Portavoz del grupo (art 110.1); 2) que sean los grupos los que tengan que sustituir a los miembros del mismo, adscritos a las diferentes comisiones (art. $40.2)$; 3) que los diputados, para cambiar de grupo, deben ser aceptados por el Portavoz del grupo donde quieren recalar (art. 26), etcétera.

En este mismo sentido, hay que indicar que la coincidencia de cargos en las estructuras orgánicas del partido y del grupo no solo contribuye a incrementar la interconexión entre ambos, sino también la coordinación y la disciplina. Principalmente, porque esto se emplea para introducir directrices partidistas

En el GPP, es el Consejo de Dirección del grupo quien establece los criterios y las directrices de actuación, mientras que es el Portavoz el encargado de decidir qué propuestas van a ser presentadas en nombre del grupo y qué diputados serán los que las defiendan. En el GPSOE, es el Comité Director quien dirige la política del grupo, autoriza las iniciativas parlamentarias y decide qué diputados las presentarán. 
en el grupo. Tal es así, que en el PP, el Presidente del partido es también el gobernante del grupo parlamentario y el Portavoz es miembro nato de la Junta Directiva Nacional (JDN), del Comité Ejecutivo Nacional (CEN), del Comité de Dirección, de la Convención Nacional, del Congreso Nacional, además de ser una de las pocas personas que acude a las reuniones de "maitines". ${ }^{3}$ Además, en el Consejo de Dirección del grupo — que es el órgano donde se estudian las propuestas legislativas y las diversas iniciativas de los diputados antes de ser presentadas ante el Congreso-, designan a los miembros que participarán en las comisiones y adoptan las medidas relacionadas con la disciplina, en el cual participan no solo los dirigentes del grupo, sino también del partido. En el PsoE, el Presidente del GPSOE en las Cortes Generales es miembro nato del Comité Federal (CF) y acude a las reuniones de la Comisión Ejecutiva Federal (CEF), con voz pero sin voto, cuando desempeña las funciones de Portavoz del grupo.

Habida cuenta de todo lo expuesto, se podría decir que la posición de los diputados en la Cámara está decidida con antelación por los órganos de gobierno del grupo. La capacidad de control es tal que los parlamentarios únicamente pueden hablar libremente en los "turnos por alusiones". ${ }^{4}$

Aunque, de todas formas, los congresistas también puedan actuar al margen del grupo parlamentario cuando solicitan información adicional en las comparecencias del Gobierno en la Cámara o cuando las preguntas quedan totalmente reservadas a los parlamentarios (art. 185, RCD); aunque, en ese caso, existe como inconveniente el número limitado de éstas, que corresponde a cada grupo y su distribución por la dirección del grupo.

En los sistemas parlamentarios actuales existe lo que se ha denominado "la disciplina del voto" con el fin de coordinar la acción y la estrategia del grupo, que se ha convertido en una práctica habitual dentro de los parlamentos (Caballero 2007), principalmente como consecuencia de las circunstancias particulares del sistema de partidos, la cohesión o centralidad organizativa de las instituciones políticas, la influencia recíproca del propio sistema político (ya sea parlamentario o presidencialista) y la vigencia de instrumentos para garantizar la disciplina de los mismos en el seno de los parlamentos.

El Estatuto del Partido Socialista Obrero Español (EPSOE) establece, en este sentido, que todos los miembros del grupo parlamentario están sujetos a la unidad de actuación y disciplina de voto (art.77) y, solo en determinados casos, el Pleno del grupo, una vez oídas las razones expuestas por los diputados, podrá acordar la libertad de voto por razones de conciencia (art. 34. RGPSOE), pero esta posibilidad se encuentra muy restringida. Además, el RGPSOE no hace más que reiterar el mandato estatutario para obligar a sus miembros a asumir los

3 Después de las reuniones de "maitines", que son unas reuniones informales de dirección del partido, el Portavoz del grupo parlamentario convoca la reunión semanal del Consejo de Dirección del grupo para organizar las actividades semanales.

4 Es cuando, en un debate, un parlamentario se refiere a otro sin que tal referencia sea el tema central del debate; y el aludido puede responder sin que medie participación de su grupo (López Aguilar 1991: 209). 
acuerdos adoptados por el grupo (art 17). En el RGPP, en tanto, se hace referencia al deber de defender los principios y las líneas básicas del programa electoral.

Aunque, pese a estos dictados, la disciplina del voto no es una obligación, sino una opción que toman los diputados para no ser sancionados y mantener su escaño en la cámara y, por eso, en la mayoría de los casos, su voto coincide con lo decidido en el Pleno del grupo. Valga de ejemplo, el episodio de indisciplina protagonizado por 15 diputados del GPSOE al negarse de abstenerse en la segunda votación de la sesión de investidura como Presidente de Mariano Rajoy. La consecuencia de dicha decisión fue una multa de $600 €$ a todos aquellos que decidieron ignorar la orden del partido de prescindir de sufragar; pero, además, algunos fueron relevados de sus cargos en el partido y en el parlamento. Esto implica que los grupos parlamentarios españoles cuenten con un alto grado de disciplina, la que solo se rompe en contadas ocasiones, dada la implicancia que tiene para los diputados que deciden no acatarla, como se ha podido observar.

\section{VII.EL RÉGIMEN DISCIPLINARIO DE LOS DIPUTADOS NACIONALES Y SUS SANCIONES}

La disciplina de los diputados abarca todo un amplio régimen disciplinario que aparece claramente expuesto en los estatutos de los partidos y en los reglamentos de los grupos parlamentarios. No obstante, no todos los representantes están sometidos a este doble sistema sancionador, ya que los parlamentarios independientes únicamente tienen que responder de sus acciones ante los órganos de gobierno del grupo. Aunque hay que advertir que también existe todo un régimen disciplinario informal que no aparece reconocido en ninguna normativa.

\section{El proceso de confección de candidaturas electorales y la reelección de los diputados}

La disciplina partidista en la Cámara se puede mantener en virtud de la confección de las listas electorales (Depauw y Martin 2009) y las características del propio sistema de elección (Carey y Shugart 1995; Bowler et al. 1999; Carey 2007). Así, por ejemplo, si nos topamos ante un sistema de listas cerradas y bloqueadas "la omnipotencia del partido queda sin ningún contrapeso eficaz" (García Canales 1995), ya que sus direcciones nacionales pasan a tener la última palabra sobre la elaboración de las listas electorales.

El estudio de los efectos de la selección de candidatos sigue estando poco desarrollado (Gallagher y Marsh 1988; Depauw y Martin 2009), tal vez porque los procesos de selección de candidatos son complejos y suelen ser resultado de las normas internas y de negociaciones informales (Hopkin 2001). 
No obstante, el control sobre el proceso de selección de los candidatos es una de las herramientas más poderosas que poseen los partidos para mantener la cohesión interna dentro su grupo parlamentario (Gallagher y Marsh 1988; Carey 2007). Por tal motivo, es imprescindible realizar un estudio que vaya más allá de las normas formales, aunque obviamente deba hacerse mención a las mismas.

En primer lugar, se han analizado las cabezas de listas en cada circunscripción, a fin de averiguar si estas tienen o no una vinculación con el ámbito territorial que representan y poder así evaluar la incidencia de la disciplina del partido. A tal efecto, se parte de la siguiente premisa: cuando existe un porcentaje alto de cabezas de listas que no tienen ninguna vinculación con la circunscripción a la que representan, se puede sostener que el partido cuenta con un alto grado de centralidad y, por tanto, el candidato ha sido impuesto por la dirección nacional y viceversa. En este sentido, en los dos partidos que son objeto de estudio, los aspirantes que encabezan las listas electorales sin tener ninguna vinculación con la circunscripción por la que fueron elegidos comienzan a tener cierta relevancia a partir de la legislatura de 2004. A partir de entonces, los postulantes que son impuestos por la cúpula de los partidos en las cabezas de las listas llegan superar el 30\%, aunque en ningún caso han alcanzado la cifra del $40 \%$. Esto significa que existe cierta discrecionalidad en la elaboración de las listas electorales, aunque se suele respetar la voluntad de las direcciones territoriales porque, de lo contrario, este porcentaje sería mayor. Pero tampoco se puede obviar que estas últimas siguen las mismas pautas que la ejecutiva nacional a la hora de elaborar las listas, con lo cual cabe pensar que, en estos ámbitos, también existe un alto grado de centralidad.

Tabla 5. Vinculación de las cabezas de las circunscripciones

\begin{tabular}{|c|c|c|c|c|c|c|c|c|c|c|c|c|c|c|c|}
\hline & \multirow{2}{*}{ Leg. } & \multicolumn{2}{|c|}{$1989 / 93$} & \multicolumn{2}{|c|}{$1993 / 96$} & \multicolumn{2}{|c|}{$1996 / 00$} & \multicolumn{2}{|c|}{$2000 / 04$} & \multicolumn{2}{|c|}{$2004 / 08$} & \multicolumn{2}{|c|}{ 2008/11 } & \multicolumn{2}{|c|}{$2011 / 15$} \\
\hline & & Frec & $\%$ & Frec & $\%$ & Frec & $\%$ & Frec & $\%$ & Frec & $\%$ & Frec & $\%$ & Frec & $\%$ \\
\hline \multirow{4}{*}{ ज̂ } & Vinculados & 32 & 61,6 & 29 & 55,8 & 35 & 67,3 & 42 & 80,8 & 32 & 61,5 & 36 & 69,3 & 36 & 69,2 \\
\hline & $\begin{array}{l}\text { No } \\
\text { vinculados }\end{array}$ & 10 & 19,2 & 15 & 28,8 & 11 & 21,2 & 10 & 19,2 & 17 & 32,7 & 15 & 28,8 & 16 & 30,8 \\
\hline & NS & 10 & 19,2 & 8 & 15,4 & 6 & 11,5 & & & 3 & 5,8 & 1 & 1,9 & & \\
\hline & Total & 52 & 100 & 52 & 100 & 52 & 100 & 52 & 100 & 52 & 100 & 52 & 100 & 52 & 100 \\
\hline \multirow{4}{*}{ 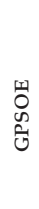 } & Vinculados & 26 & 50 & 33 & 64,5 & 37 & 71,2 & 41 & 78,8 & 35 & 67,3 & 31 & 59,6 & 34 & 65,4 \\
\hline & $\begin{array}{l}\text { No } \\
\text { vinculados }\end{array}$ & 18 & 34,6 & 14 & 26,9 & 9 & 17,3 & 9 & 17,4 & 15 & 28,4 & 20 & 38,5 & 17 & 32,7 \\
\hline & NS & 8 & 15,4 & 5 & 9,5 & 6 & 11,5 & 2 & 3,8 & 2 & 3,8 & 1 & 1,9 & 1 & 1,9 \\
\hline & Total & 52 & 100 & 52 & 100 & 52 & 100 & 52 & 100 & 52 & 100 & 52 & 100 & 52 & 100 \\
\hline
\end{tabular}

Fuente: elaboración propia, tomando la base de datos del Congreso de los Diputados y el Boletín Oficial del Estado

Otra cuestión es el sistema de selección para elegir a los candidatos. Habitualmente, en todos los partidos existe un Comité o Comisión Electoral que se encarga de elaborar las listas en función de los informes que les presentan otros órganos del partido y sus homólogos territoriales. Sin embargo, en otros 
casos, únicamente se limitan a aprobar las propuestas que son enviadas desde las organizaciones regionales y provinciales aunque, en la práctica, la opinión de los órganos inferiores es respetada en la medida en que coincide con los intereses de los dirigentes nacionales (Flores 1998: 228).

Los estatutos del pP son claros a este respecto: corresponde al Comité Electoral Nacional ratificar las candidaturas de las elecciones legislativas. Las listas electorales han sido elaboradas desde 1979 por las organizaciones territoriales correspondientes, aunque siempre ha existido un órgano en la estructura central del partido con capacidad para ratificar o modificar las propuestas que les eran presentadas (García-Guereta 2001: 485). Por tanto, la última palabra sobre las mismas siempre la tiene la dirigenza gracias al Comité Electoral Nacional del partido, cuyos miembros son nombrados por su Comité Ejecutivo.

En el PsOe, es el Comité Federal (CF) del partido el encargado de ratificar las propuestas que le son presentadas por la Comisión de Listas que, por otra parte, él mismo ha nombrado en su primera reunión después del Congreso Federal. Además, el Comité Electoral es el que establece los criterios de selección, designa a la mayoría de los miembros de la Comisión Federal de Listas, nombra al candidato a la Presidencia del Gobierno y decide sobre cualquier acuerdo pre o postelectoral con otras fuerzas políticas, así como sobre la celebración de primarias; mientras que la Comisión Ejecutiva Federal (CEF) elabora el calendario a seguir para elegir a los aspirantes, nombrando a parte de los miembros de la Comisión de Listas y, en caso de llevarse a cabo las elecciones primarias, proclama al contendor a la Presidencia del Gobierno.

Tabla 6. Índice de centralidad y exclusividad en la selección de los diputados

\begin{tabular}{lrrr}
\hline & $\begin{array}{c}\text { Índice de } \\
\text { centralidad } \\
(0-14)\end{array}$ & $\begin{array}{c}\text { Índice de } \\
\text { exclusividad } \\
(1-100)\end{array}$ & $\begin{array}{c}\mathrm{N}^{\mathrm{o}} \text { de } \\
\text { diputados } \\
\text { (N de grupos) }\end{array}$ \\
\hline Diputados nacionales del PP & 9,8 & 77,5 & $51(2)$ \\
Diputados nacionales del PSOE & 11,9 & 59,4 & $55(2)$ \\
$\begin{array}{l}\text { Diputados nacionales de otros } \\
\text { partidos }\end{array}$ & 11,0 & 85,4 & $27(5)$ \\
Media & 11,0 & 68,1 & $133(9)$ \\
\hline
\end{tabular}

Fuente: Cordero y Coller (2013: 23)

Ahora bien, hasta aquí se ha hecho alusión a la normativa vigente pero, para poder continuar midiendo el grado de centralidad del proceso —e ir más allá-, hay preguntarse: ¿cuál es el verdadero papel que desempeñan los distintos actores (nacionales, regionales y locales) en el proceso de selección de candidatos? (Norris y Lovendusky 1995). La percepción que tienen los diputados sobre el proceso de selección es que este se encuentra altamente centralizado, más en el caso del PSOE y de otros partidos que en el del Pr. No obstante, la media de la 
Tabla 6 fue de once sobre 14 y, por tanto, no es de extrañar la propensión que muestran los parlamentarios a la hora de seguir la línea del grupo (Bowler et al. 1999). Como el proceso es controlado por el centro, el partido puede asegurar la cohesión del cuerpo legislativo (Gallagher y Marsh 1988).

Otra cuestión que también se debe analizar es el índice de exclusividad. Para ello hay que responder a esta pregunta, ¿cómo fue su elección para ir en las listas? El promedio de las respuestas fue de 69,2 en una escala del 1 a 100, lo que implica que la percepción de los diputados se sitúa cerca del polo de exclusividad (Cordero y Coller 2013: 23), principalmente por la lealtad que muestran ante el líder que los ha propuesto y gracias al sistema electoral imperante.

Para complementar esta información, habría que conocer cuáles son los factores que determinan la incorporación de los candidatos en las listas electorales. Según los datos de la Tabla 7, los tres criterios informales más importantes para escoger a los futuros parlamentarios son: el conocimiento de los problemas, la dedicación y la lealtad, aspectos que se encuentran vinculados a la vida interna de los partidos (Helmke y Levitsky 2004). A continuación le siguen: la preparación y la experiencia, que son elementos relacionados con la capacidad de los diputados, mientras que los demás criterios apenas tienen relevancia. Esto indica que existe cierta discrecionalidad en este proceso en todas las fuerzas políticas.

\section{Tabla 7. Causas percibidas de la selección de candidatos}

\begin{tabular}{lrrr}
\hline & PP & PSOE & \multicolumn{1}{l}{ IU } \\
\hline Conocimiento problemas & 4,1 & 4,1 & 4,3 \\
Dedicación & 4,0 & 4,0 & 4,1 \\
Lealtad & 4,0 & 3,8 & 3,7 \\
Preparación & 3,8 & 3,8 & 3,3 \\
Experiencia & 3,9 & 3,6 & 3,3 \\
Prestigio & 3,6 & 3,6 & 3,3 \\
Apoyo líder & 3,4 & 3,4 & 2,9 \\
Org. local del partido & 2,8 & 3,0 & 2,5 \\
Org. provincial partido & 2,6 & 2,7 & 2,6 \\
Familia & 1,5 & 1,4 & 1,4 \\
Otras & 4,5 & 4,5 & 4,0 \\
\hline
\end{tabular}

Fuente: base de datos del Centro de Investigaciones Sociológicas (cIs), Estudio 2827

Además, estos datos hay que vincularlos con la opinión de los diputados sobre este proceso. En este sentido, estos consideran que el sistema de selección gira en torno a dos cuestiones: los más preparados y la lealtad, después le siguen los más populares y los mejores (ver Tabla 8). Por tanto, existe una tensión entre lealtad y preparación, aunque la balanza parece decantar — en todas las fuerzas 
políticas - por lealtad y dedicación, y eso, indiscutiblemente, repercute en la cohesión de los grupos parlamentarios.

Tabla 8. Opinión sobre la selección

\begin{tabular}{lrrr}
\hline & PP & PSOE & IU \\
\hline Se pretende buscar a... & & & \\
Los mejores & 4,8 & 4,4 & 4,1 \\
Los más leales & 5,1 & 4,9 & 4,1 \\
Los más expertos & 4,5 & 4,0 & 3,2 \\
Los más experimentados & 4,6 & 4,4 & 4,0 \\
Los más populares & 4,8 & 4,4 & 4,1 \\
Los más preparados & 5,2 & 4,7 & 4,7 \\
\hline
\end{tabular}

Fuente: base de datos del Centro de Investigaciones Sociológicas (CIS), Estudio 2827

Así, observamos que el grado de poder que tienen las cúpulas de los partidos políticos en este proceso es casi absoluto. Además, el hecho de que España todavía cuente con un sistema de listas cerradas y bloqueadas no viene más que a reforzar este hecho. Si se tuvieran listas abiertas, o al menos cerradas pero desbloqueadas, la incidencia de los electores en este proceso sería mayor y, por tanto, esto repercutiría positivamente en el grado de autonomía que dispondrían los parlamentarios para poder ejercer sus funciones.

\section{Renovación de los grupos parlamentarios}

Para medir la disciplina del partido también es necesario evaluar el grado de renovación de los grupos parlamentarios. Para analizar este aspecto, se tomará como referencia la cifra del 70\% de permanencia de los diputados de una legislatura a otra (Sánchez de Dios 1996: 8). Ello debido a que el nivel de experiencia que poseen los congresistas puede influir en el grado de disciplina y también porque cuando mantienen una actitud díscola, se produce una alta renovación del grupo para sustituir aquellos que no se someten a la disciplina de este.

En la Tabla 9, tanto el GPP como el GPSOE son grupos parlamentarios muy estables. Únicamente en la IV legislatura (1989/93) y en la X (2011/2015), los diputados del GPP sufrieron una renovación que alcanzó el 65,11\% y 50,5\%, respectivamente. Datos que obedecen a dos factores externos más que a la propia merma del grado de disciplina interna. En primer lugar, en 1989 el PP se encuentra sumergido en un proceso de refundación del partido, que indudablemente origina un cambio en la élite dirigente del partido. En segundo término, en 2011 se logran los mejores resultados electorales de su historia y, por tanto, el grupo parlamentario incrementa su presencia en el Congreso con 
26 escaños, derivándose de ello una significativa incidencia en el grado de renovación del grupo por la vía del crecimiento y de las nuevas incorporaciones. En el GPSOE se produce algo similar, superando solamente la renovación del 50\% en 2000 y 2004, como consecuencia de un cambio de liderazgo en el primer caso y un incremento del número de diputados, (37) en el segundo.

Tabla 9. Renovación de los diputados nacionales (1989/2015)

\begin{tabular}{|c|c|c|c|c|c|c|c|c|c|c|c|c|c|c|c|}
\hline & \multirow{2}{*}{ Leg. } & \multicolumn{2}{|c|}{$1989 / 93$} & \multicolumn{2}{|c|}{$1993 / 96$} & \multicolumn{2}{|c|}{$1996 / 00$} & \multicolumn{2}{|c|}{$2000 / 04$} & \multicolumn{2}{|c|}{ 2004/08 } & \multicolumn{2}{|c|}{ 2008/11 } & \multicolumn{2}{|c|}{$2011 / 15$} \\
\hline & & Frec. & $\%$ & Frec. & $\%$ & Frec. & $\%$ & Frec. & $\%$ & Frec. & $\%$ & Frec. & $\%$ & Frec. & $\%$ \\
\hline \multirow{3}{*}{ ثิ } & Reelegidos & 37 & 34,9 & 71 & 50,3 & 92 & 59,3 & 95 & 53,1 & 92 & 62,2 & 84 & 54,5 & 92 & 49,5 \\
\hline & $\begin{array}{l}\text { Diputados } \\
\text { nuevos }\end{array}$ & 69 & 65,1 & 70 & 49,3 & 62 & 40,3 & 84 & 46,9 & 56 & 37,8 & 70 & 45,5 & 94 & 50,5 \\
\hline & Total & 106 & 100 & 141 & 100 & 154 & 100 & 179 & 100 & 148 & 100 & 154 & 100 & 186 & 100 \\
\hline \multirow{3}{*}{$\begin{array}{l}\text { II } \\
0 \\
\text { t. } \\
\text { th }\end{array}$} & Reelegidos & 114 & 65,2 & 105 & 66 & 75 & 53,2 & 61 & 48,8 & 61 & 37,7 & 91 & 53,8 & 86 & 78,2 \\
\hline & $\begin{array}{l}\text { Diputados } \\
\text { nuevos }\end{array}$ & 61 & 34,8 & 54 & 34 & 66 & 46,8 & 64 & 51,2 & 101 & 62,3 & 78 & 46,2 & 24 & 21,8 \\
\hline & Total & 175 & 100 & 159 & 100 & 141 & 100 & 125 & 100 & 162 & 100 & 169 & 100 & 110 & 100 \\
\hline
\end{tabular}

Fuente: elaboración propia, con la base de datos del Congreso de los Diputados

\section{El voto secreto, libertad de voto y la asistencia obligatoria a las sesiones}

El voto secreto permite burlar la disciplina partidista, ya que, al no identificarse el voto con la persona, los diputados pueden expresar su opinión libremente y no en función de la orientación que ha fijado su grupo parlamentario. Tal vez por este motivo, el voto secreto haya sido escasamente utilizado, máxime cuando su mera solicitud puede ser sancionada por la dirección del partido y del grupo parlamentario. Además, la votación secreta queda totalmente excluida para los procedimientos legislativos, las sesiones de investidura, las mociones de censura, la cuestión de confianza y los acuerdos que hayan de adoptarse en función del criterio de voto ponderado. Esto implica que la unidad de voto se encuentra condicionada por el control que ejerce el grupo y por los incentivos positivos y negativos que éste ofrece a sus diputados (Hechter 1983).

En este sentido, cabe decir que, por ejemplo, para no poder burlar la disciplina de voto, todos los reglamentos de los grupos exigen a sus miembros la asistencia a las reuniones de los plenos, comisiones, ponencias y votaciones, al tiempo que imponen una serie de sanciones ante ausencias no autorizadas. Esta es la manera que han encontrado los grupos para que los parlamentarios no violen la disciplina mediante el recurso de ausentarse. Tal es así que, en el GPSOE, es el Comité Director del grupo quien elabora el informe de las inasistencias para que el Comité de Disciplina sancione a los diputados por las ausencias injustificadas. En el GPP solo se penalizan las ausencias en el Pleno de la cámara, y no en las Comisiones. 
No obstante, y pese a estas disposiciones, los diputados pueden negarse a acatar estas sanciones, otra cosa es que estén dispuestos a hacerlo ante los altos costes políticos que podrían ocasionarles tal decisión. Incluso en el RCD se considera que la asistencia al Pleno y a las comisiones es un deber con el que tienen que cumplir los diputados electos (art. 15 RCD) y, para ello, la Mesa del Congreso sanciona a aquellos parlamentarios que dejen de acudir de forma reiterada o notoria a las sesiones del Pleno o de las Comisiones (art. 99. RCD).

\section{Recompensas y devolución o renuncia al acta parlamentaria}

Las medidas disciplinarias informales son: las recompensas ligadas, por ejemplo, a la participación en las comisiones más relevantes; la concesión de más recursos para desempeñar sus trabajos, o la defensa de las iniciativas o propuestas más importantes. Las direcciones de los grupos suelen premiar a los diputados que se comportan coherentemente (Longley y Hazan 1999) y, gracias a ello, éstos pueden refuerzan su carrera política, mientras que los más díscolos ven como su proyección política queda estancada o bloqueada al ser relegados a las comisiones menos relevantes, al contar con menos recursos y al negarles la posibilidad de presentar sus iniciativas o propuestas (Russell 2008).

Otro mecanismo sancionador que no aparece recogido en los reglamentos de los grupos parlamentarios es la renuncia en blanco de los diputados. En principio, ésta se hace sin fecha y el partido se convierte en el depositario y transmisor de una declaración de voluntad ajena, previamente realizada, que deja bajo su custodia (De Vega 1985: 43). A priori se presupone que los parlamentarios la firman libremente, pero, en realidad, se trata de una exigencia que los partidos imponen a sus candidatos para ser incluidos en las listas electorales. No obstante, los diputados pueden acudir a los tribunales para que esta renuncia por escrito no se haga efectiva en virtud de la sentencia 5/1983 del Tribunal Constitucional, del 4 de febrero, en la que se reconoce el derecho a acceder a los cargos públicos y que comprende también el derecho a permanecer en los mismos, porque de otro modo, el derecho fundamental quedaría vacío de contenido. Por tanto, esta sentencia desautoriza a los partidos para hacer valer este tipo de documentos si el diputado se manifiesta en contra. De ahí que la Mesa del Congreso, como receptora de las renuncias, tenga que comprobar si la voluntad recogida en el documento de dimisión que se le presenta resulta adecuada a los auténticos deseos del diputado (Morales 1990: 301).

\section{CONCLUSIONES}

Este artículo se planteaba como objetivo medir el grado de disciplina de los grupos parlamentarios en la Cámara Baja, de manera que se pudiera determinar cuál es su naturaleza y las causas y las consecuencias de la misma. En este 
sentido, cabe decir que España evidencia un alto grado de dependencia de los grupos parlamentarios y los diputados sobre el partido.

Nos encontramos ante una "parlamentarización de los partidos" (Agh y Sandor 1995: 251), y esto solo es posible porque la normativa de los partidos, la Constitución, el RCD, los reglamentos internos de los grupos parlamentarios y el sistema electoral han dado cabida a esta nueva realidad, reconociendo abiertamente el papel predominante de los partidos en el funcionamiento del parlamento, arruinando con ello el clásico mandato representativo del parlamentario individual. Ello solo logra reestablecerse cuando los diputados consideran que es más beneficioso seguir la opinión de los electores que la del partido, porque son los votantes quienes tienen el poder real para garantizar su reelección (Bowler y Farrell 1993) o porque no desean continuar con su carrera parlamentaria, lo que les permite tener una gran autonomía de actuación.

No obstante, es cierto que - a priori- los diputados tienen derecho a ejercer con plena libertad todas las facultades constitutivas de su ius in officium que les otorga el ordenamiento jurídico y reglamentario, pero la realidad es que el parlamentario puede ejercer por sí un número reducido de competencias, formando un catálogo menor de funciones y de escaso contenido e importancia política. Es cierto que la disciplina es necesaria para garantizar el funcionamiento del sistema democrático y no caer en un bloqueo institucional que produzca un impasse político. El problema es que los partidos políticos abusan de la misma, mientras que la autonomía de los diputados queda prácticamente reservada al quehacer diario y a las épocas de conflictividad interna en los partidos políticos o cuando los grupos parlamentarios se encuentran formados por varias fuerzas políticas de diferentes intereses. Por tanto, lo ideal sería buscar un equilibrio (que todavía no se ha encontrado) entre la disciplina y la autonomía.

En todo caso, el grado de disciplina se encuentra vinculado a varios factores, como se ha podido comprobar a lo largo de este trabajo. En primer lugar, la dirección de los partidos controla la mayor parte de los espacios de los grupos parlamentarios, gracias a que designa a sus órganos de dirección que son, en definitiva, los que marcan las pautas de actuación del mismo y de sus miembros. Además, se produce un sistema de comunicación bidireccional, en virtud a que cargos del partido forman parte de los órganos de dirección del grupo y viceversa. Tal es así que el $60 \%$ de las decisiones que se adoptan en los grupos parlamentarios son consecuencia de la propia disciplina del partido, mientras que solo una pequeña fracción son fruto de la deliberación. Esto no es un hecho aislado de España, sino que también se produce en una gran parte de las democracias europeas, lo que se demuestra en el índice Rice que arrojan estas. Evidentemente, esto no tienen la misma incidencia si se trata de un grupo numeroso o pequeño, o si los electores tienen o no capacidad para determinar la composición de la cámara.

En segundo término, la elaboración de las listas electorales está altamente centralizada por la cúpula nacional del partido, gracias, en parte, al sistema 
electoral y las normas estatutarias imperantes. Como consecuencia de ello, el índice de centralidad y exclusividad en la selección de los diputados se sitúan en cifras elevadas. De ahí que criterios detectados para forma parte de las listas electorales sean totalmente informales, lo que ahonda en el alto grado de discrecionalidad que viene a favorecer la disciplina parlamentaria, más cuando los votantes no poseen la capacidad para poder influir en la composición de la Cámara.

Comotercer factor seencuentra elgradoderenovación delasélites parlamentarias, el cual también incide en la disciplina dentro de los grupos parlamentarios. El porcentaje de permanencia de los diputados implica el sometimiento de estos a la disciplina del grupo, porque cuando existe indisciplina se produce un alto porcentaje de renovación de los mismos.

Y por último, la indisciplina de los parlamentarios tiene un alto coste para aquellos que deseen hacer carrera política. Primero, porque si rompen la conducta del voto son sancionados económicamente y, de mantener esta actitud, no serán incluidos en las listas en unas futuras elecciones, incluso pueden ser obligados a dimitir gracias a la renuncia en blanco que firman antes de ser integrados en las listas electorales. Asimismo, porque los diputados que no siguen con la línea marcada por la dirección del grupo ven cómo queda estancada o bloqueada su carrera política. Medidas disciplinarias como estas que no aparecen, en muchos de los casos, recogidas en la normativa de los partidos.

\section{REFERENCIAS}

Agh, Attila, y Kurtan Sandor. 1995. Democratization and Europeanization in Hungary: The First Parliament, 1990-1994. Budapest: Hungarian Centre for Democratic Studies.

Aragón Reyes, Manuel. 2008. "Democracia y parlamento." Revista Catalana de Dret Públic 37: 129-155.

Arter, David. 1984. The Nordic Parliaments: A Comparative Analysis. London: Hurst.

Béjar Algazi, Luisa. 2002. "El marco institucional de la disciplina parlamentaria en México." En Política en América Latina, editado por Manuel Alcántara. Salamanca: Universidad de Salamanca, 2530-2552.

Bowler, Shaun, y David M. Farrell. 1993. "Legislator Shirking and Voter Monitoring: Impacts of European Parliament Electoral Systems Upon Legislator-Voter Relationships." Journal of Common Market Studies 31 (1): 45-69.

Bowler, Shaun; David M. Farrell y Richard S. Katz. 1999. "Party Cohesion, Party Discipline and Parliaments." En Party Discipline and Parliamentary Government, editado por Shaun Bowler, David M. Farrell y Richard S. Katz. Columbus: Ohio State University Press, 3-22.

Caballero, Gonzalo. 2007. "Comisiones, grupos parlamentarios y diputados en la gobernanza del Congreso de los Diputados." Revista de Estudios Políticos 135: 67-107.

Carey, John M. 2000. "Party Unity in Legislative Voting." Ponencia presentada en American Political Science Associations Conference, Washington, 5 de septiembre, 1-32.

Carey, John M. 2007. “Competing Principals, Political Institutions and Party Unity in Legislative Voting." American Journal of Political Science 51 (1): 92-107.

Carey, John, y Matthew Shugart. 1995. "Incentives to Cultivate a Personal Vote: A Rank Ordering of Electoral Formulas." Electoral Studies 14(4): 417-438. 
Cordero, Guillermo, y Xavier Coller. 2013. “Candidate Selection and Party Discipline." Ponencia presentada en ECPR General Conference, Sciences Po Bordeaux, 4 al 7 de septiembre: $1-26$.

Cox, Gary; Mathew D. McCubbins y Adriana Prata. 2009. Setting the Agenda: Parliaments, Procedural Cartels and Policy Making. Nueva York: Cambridge University Press.

Damgaard, Erik. 1992. Parliamentary Change in the Nordic Countries. Oslo: Scandinavian University Press.

De Vega, Pedro. 1985. "Significado constitucional de la representación política." Revista de Estudios Políticos 44: 25-44.

Depauw, Sam, y Shane Martin. 2009. “Legislative Party Discipline and Cohesion in Comparative Perspective." En Intra-Party Politics and Coalition Governments, editado por Daniela Giannetti y Kenneth Benoit. Londres: Taylor and Francis, 1-30.

Field, Bonnie N. (2016). Why Minority Governments Work: Multilevel Territorial Politics in Spain. Nueva York: Palgrave Macmillan.

Flores Giménez, Fernando. 1998. La democracia interna de los partidos políticos. Madrid: Congreso de los Diputados.

Gallagher, Michael, y Michael Marsh. 1998. Candidate Selection in Comparative Perspective: The Secret Garden of Politics. Londres: Sage.

García Canales, Mariano. 1995. "La huella presidencialista en el constitucionalismo español." Revista Española de Derecho Constitucional 44: 120-127.

García-Guereta Rodríguez, Elena María. 2001. "Factores externos e internos en la transformación de los partidos políticos. El caso de AP-PP". Ph.D. Madrid: Instituto Juan March de Estudios e Investigaciones.

González Tule, Luis A. 2010. “Disciplina parlamentaria en América Latina en opinión de los legisladores." Boletín Datos de Opinión 16: 1-4.

Guerrero Salom, Enrique. 2004. El Parlamento. Qué es, cómo, funciona, qué hace. Madrid: Editorial Síntesis.

Hazan, Reuven Y. 2003. "Does Cohesion Equal Discipline? Towards a Conceptual Delineation." Journal of Legislative Studies 9 (4): 1-11.

Hechter, Michael. 1983. "A Theory of Group Solidarity". En Microfoundations of Macrosociology, editado por Michael Hechter. Filadelfia: Temple University Press, 16-57.

Heller, William, y Carol Mershon. 2002. “Dealing Discipline: Party Switching and Legislative Voting in the Italian Chamber of Deputies, 1996-2000." Ponencia presentada en Annual Meetings of the Midwest Political Science Association, Chicago, 25 al 28 de abril, 1-30.

Helmke, Gretchen, y Steven Levitsky. 2004. "Informal Institutions and Comparative Politics: A Research Agenda." Perspectives on Politics 2 (4): 725-740.

Holgado González, María. 2016. “¿Pueden los parlamentarios votar libremente?” Revista de Faculdade de Direito 2 (61): 9-23.

Hopkin, Jonathan. 2001. "Bringing the Members Back In? Democratizing Candidate Selection in Britain and Spain." Party Politics 7 (3): 343-361.

Lacina, Tomas. 2009. "Legislative Roll Call Behaviour in the Czech Republic." Ponencia presentada en ECPR, Lisboa (Portugal), 14-19 de abril, 1-36.

Laver, Michael, y Kenneth Shepsle. 1999. "How Political Parties Emerged from the Primeval Slime: Party Discipline, Intraparty Politics and Government Formation." En Party Cohesion, Party Discipline and the Organization of Parliaments, editado por S. Bowler, Shaun, David M. Farrel y Richard S. Katz. Columbus: Ohio State University Press, 23-48.

Lees, John, y Malcolm Shaw. 1979. Committees in Legislatures: A Comparative Analysis. Durham: Duke University Press.

Longley, Lawrence D., y Reuven Y. Hazan. 1999. “On the Uneasy, Delicate, yet Necessary Relationships between Parliamentary Members and Leaders." Journal of Legislative Studies 5 (3-4): 1-22.

López Aguilar, Juan Fernando. 1991. Minoría y oposición en el parlamentarismo. Una aproximación comparativa. Madrid: Congreso de los Diputados. 
Morales Arroyo, José M. 1990. Los grupos parlamentarios en las Cortes Generales. Madrid: Centro de Estudios Constitucionales.

Nacif, Benito. 2002. "Para comprender la disciplina de partido en la Cámara de Diputados de México. El modelo de partido centralizado." Foro Internacional 42 (1): 5-38.

Norris, Pippa, y Joni Lovendusky. 1995. Political Recruitment: Gender, Race and Class in the British Parliament. Cambridge: Cambridge University Press.

Ozbudun, Ergun. 1970. Party Cohesion in Western Democracies: A Causal Analysis. Beverly Hills: Sage Books.

Raunio, Tapio. 2000. "Second-Rate Parties? Towards a Better Understanding of European Parliament's Party Groups." En Parliamentary Party Groups in European Democracies: Political Parties behind Closed Doors, editado por Knut Heidar y Ruud Koole. Londres: Routledge, 233-247.

Russell, Meg. 2008. “Explaining Parliamentary Party Cohesion: Can Psychology Help?" Ponencia presentada en for International Society of Political Psychology Conference, París, 9-12 de julio, 1-26.

Saalfeld, Thomas, y Richard Becker. 2000. "Cohesion, Veto Players and Agenda Control: Explaining the Dynamics of the Legislative Process in 17 West European Parliaments." Ponencia presentada en uK Political Studies Association, Londres, 10-13 de abril, 1-27.

Sánchez de Dios, Manuel. 1996. "La disciplina de partido en los grupos parlamentarios del Congreso de los Diputados." Revista de las Cortes Generales 39: 183-210.

Sartori, Giovanni. 1999. "En defensa de la representación política." Claves de Razón Práctica 91: 2-8.

Stratmann, Thomas, y Martin Baur. 2002. "Plurality Rule, Proportional Representation and the German Bundestag: How Incentives to Pork-Barrel Differ Across Electoral Systems." American Journal of Political Science 3 (46): 506-514.

Gema Sánchez Medero es profesora de la Facultad de Ciencias Políticas y Sociología en la Universidad Complutense de Madrid, misma casa de estudios donde obtuvo su grado de Doctora en Ciencias Políticas en 2007. Anteriormente fue profesora ayudante en la Universidad Carlos III de Madrid y becaria predoctoral del Congreso de los Diputados. Es secretaria de la Revista Cuadernos de Gobierno y Administración Pública y responsable del área de formación en el Instituto Complutense de Ciencia de la Administración, ambos de la Universidad Complutense de Madrid. Participa en diversos master y cursos en la Universidad Complutense, en el Instituto Nacional de Administraciones Públicas (España), la Universidad de Alicante, la Universidad de Alcalá de Henares, el Centro Superior de Estudios de la Defensa Nacional (CESEDEN), entre otros. Asimismo, ha participado como profesora o investigadora invitada en las universidades de Florencia (Italia), de Costa Rica, La República, de Michigan y de Minnesota, además del Instituto de Altos Estudios para la Defensa de la República de Venezuela, entre otros. Correo electrónico: gsmedero@cps.ucm.es

Bernabé Aldeguer Cerdá es licenciado en ciencias políticas y de la administración por la Universidad Miguel Hernández de Elche (UMH) y doctor en derecho (estudios políticos y constitucionales) por la Universidad de Alicante (UA), galardonado con el Premio Extraordinario de Licenciatura, el Certificado de Excelencia Académica (Generalitat Valenciana) y la Mención Especial del Ministerio de Educación (Gobierno de España). En 2004 obtuvo el $1^{\circ}$ Accésit del XIII Premio de Ensayo en Ciencias Sociales "Fermín Caballero" y el Premio a la Tesis Doctoral por el Parlamento Autonómico Valenciano (Cortes Valencianas). Es autor de diversos capítulos de libro y manuales universitarios, así como distintos artículos académicos en revistas nacionales e internacionales. Correo electrónico: bernabealdeguer@gmail.com 